\title{
II.5. Confocal and multiphoton endomicroscopy
}

Claire Lefort

XLIM Research Institute, UMR CNRS 7252, Université de Limoges, France

\section{Abstract}

Endoscopic examination is a well-established medical practice and helps hospital practitioners to orientate diagnostic and treatments with a painless protocol which also contributes to improve the care of patients. But the endoscopic images generated are restrained to a surface imaging and image quality is limited to a millimetric resolution. The precision of the diagnostic can be impacted by these restrictions and some significant alteration of the structure, metabolism or physiology of organs can be missed. Today, many technical developments are in progress for the development of endomicroscopes. That imposes for example to resort to the intermediary of an optical waveguide whose internal structure imposes many technical and computational constraints, discussed in the present article. The miniaturization of distal optics or scanning devices are still in progress of elaboration simultaneously with an optimal computational strategy for erasing image defaults linked to the necessity of extremal miniaturization.

\section{Introduction}

Endoscopic examination is a routine practice striving to guide medical diagnosis, without resorting necessarily to a surgical biopsy. This medical procedure is mainly indicated for the visual exploration of the interior of organs accessible by natural paths, directly inside the patient, in vivo and in situ. Laryngoscopy for larynx exploration, bronchoscopy for lung exploration and colonoscopy for colon exploration are few 
examples of routine endoscopic practices. The main interest of an endoscopic exploration rests on its ability to substitute invasive surgical biopsies.

The resolution associated to standard endoscopes is millimetric, an insufficient range in some cases. In that context, technical researches are now focused on the development of endomicroscopic systems having a micrometer and sub-cellular resolution [1-4]. The distinction between an endoscopic and an endomicroscopic device mainly concerns ranges of resolution, field of view and process of image generation. Today, Mauna Kea Technologies ${ }^{1}$ is the international company leader in the field of confocal endomicroscopy. The final goal leading to the technological development of endomicroscopic systems is to deliver a tool generating in vivo and in situ endomicroscopic images having a quality similar to those generated by bulk optical microscopes. Thus, imaging in vivo structures with a microscopic resolution becomes possible in a minimally invasive way. The emergence of optical guides such as optical fibers, miniaturized lenses and scanning devices are associated to the highest progresses for the development of endomicroscopes. Nevertheless, numerous technical challenges are not yet enough mature and are in fact still on the spotlight in order to obtain a final tool able to generalize the routine practice of endomicroscopy into hospitals.

\section{Principles \& Set-ups}

\subsection{General presentation of an endoscopic exploration}

Three kinds of endoscopic systems are discussed in the present chapter, i.e., standard, confocal and multiphoton endoscopic systems. The standard endoscope is considered

\footnotetext{
${ }^{1}$ https://www.maunakeatech.com/int/
} 
as a reference; its practice at the hospital is now well-established and well-mastered by hospital practitioners. Its principle rests on the miniaturization of a video camera associated with a white light. An image of the surface of the interior of organs is generated with a millimetric resolution. Figure 1 illustrates schematically the endoscopic exploration of lung. Such an endoscopic system is composed by an optical tube dedicated to enter into a human cavity for its examination - here lungs - a handling segment to guide manually the optical tube and a hardware system containing the lighting and the imaging parts. The region of the endoscope the closest to the target imaged is named "distal end". Reversely, the optical tube input is the proximal section of the endoscope.

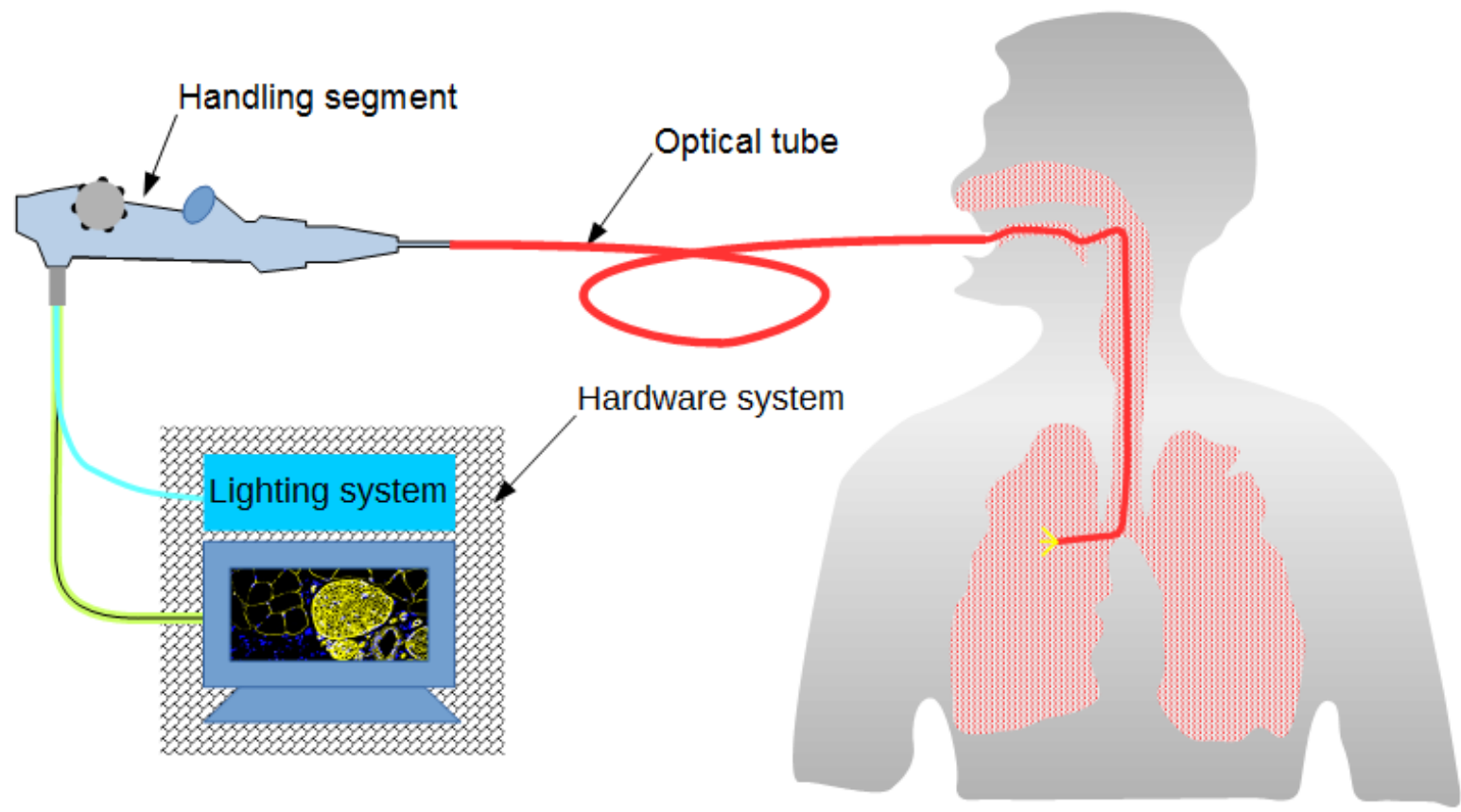

Fig. 1 Scheme illustrating an endoscopic system.

The images generated by an endoscopic system are restrained to a surface image with a millimetric resolution. The precision of the diagnostic can be impacted by these restrictions and some significant alterations of the structure, metabolism or physiology 
of organs can be missed. Thus, a clinical need to have access to an endomicroscopic system is meaningful.

\subsection{Physical Principles of an endomicroscope}

From the viewpoint of clinicians, the overall handling of an endomicroscope is identical to the handling of an endoscope. Only technical differences exist but do not impact the tool handling. Currently, confocal endomicroscopes are yet marginally implemented into hospitals and no commercial multiphoton endomicroscopic system exists yet; the technical specificities of confocal and multiphoton endomicroscopes are detailed in the following parts. Their physical principles rest mainly on the generation of fluorescence signals. Thus, identical properties of the excitation light (coherent light, wavelength ranges, average powers...) and emission signals are involved between endomicroscopes and bulk microscopes. We can notice here that harmonic generation is an alternative imaging modality that can be generated in multiphoton endomicroscopy. More details about signal generation can be found into chapter I. 1 .

b. Fluorescence \& Confocal Microscopy and into chapter I. 2. c. Multiphoton microscopy. The specificity of endomicroscopes compared to bulk microscopes rests on the miniaturization and the transfer of the excitation beam and the detection strategy in vivo and in situ, the closest to the imaged target.

\subsection{Technical principles, typical set-up \& State-of-the-art of endomicroscopy} The optical waveguide contained into the optical tube (Fig. 1), delivers the excitation beam and collects the emission signals. This element constitutes the key point of an endomicroscope. Two kinds of optical waveguides can be implemented into an endomicroscopic system: a double clad fiber (DCF) with a single core and a fiber-optic 
bundle composed by few thousands of optical cores [6, 7]. Fig. 2 presents technically the structure of the endomicroscope and the differences induced by each of the two strategies. The choice of the optical waveguide impacts the time delay for generating and image, the scanning strategy, the level of miniaturization of the distal end and the complexity of the computational strategy required for the optimization of image quality. The optical waveguide is also an element potentially responsible for self-fluorescence generating background noise. A solution of pure silica structure should be preferred in that case $[8,9]$. Furthermore, whatever the optical waveguide chosen, a focusing element must be positioned at the endoscopic distal end.

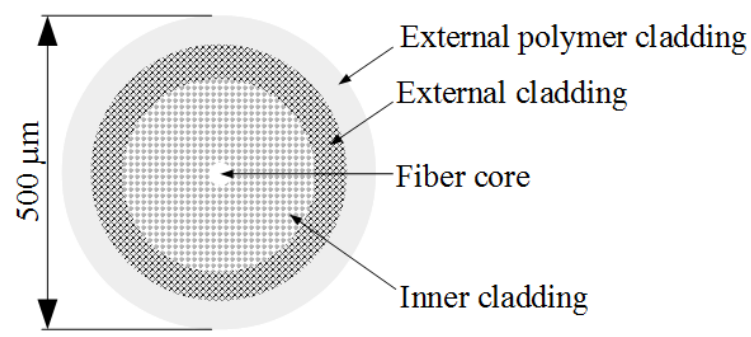

$\underline{\text { Double clad fiber }}$

Examined organ

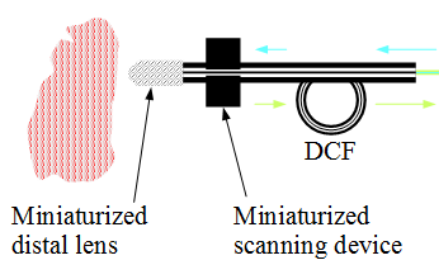

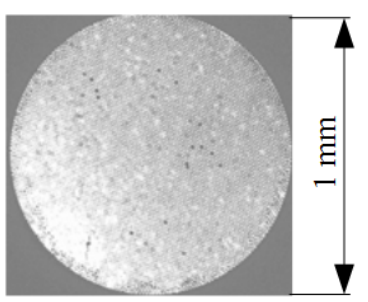

Fiber-optics bundle

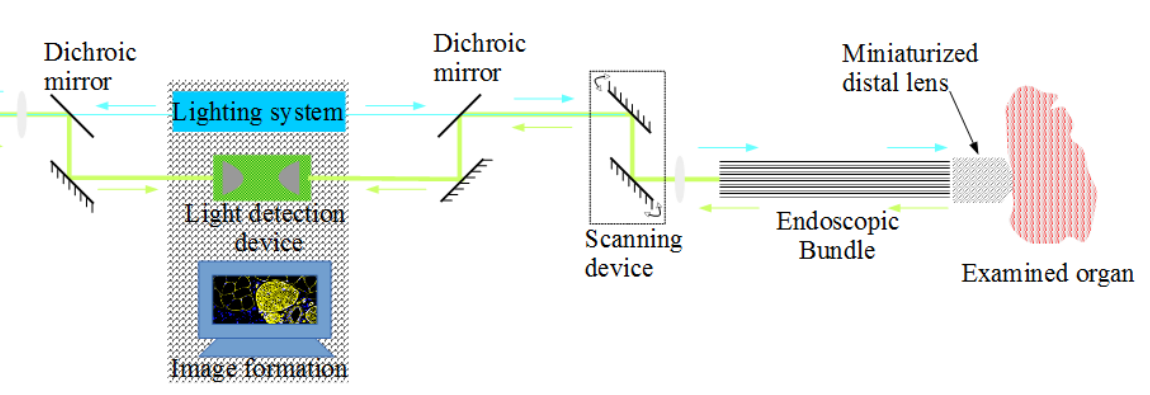

Fig. 2. Presentation of the two architectures of an endomicroscope depending on the optical waveguide chosen. A double clad fiber (DCF, left) and a fiber-optics bundle (right) are the two technical strategies that can be encountered into an endomicroscopic system.

\section{Focusing element at endoscopic distal end}

Regardless of the optical waveguide chosen, reaching microscopic performances of an endomicroscopic system requires the use of a miniaturized focusing element positioned at endoscopic distal end. This optical microsystem governs the optical 
resolution, the image field of view and working distance. The design of the dimensions and optical properties of the focusing element can be modeled numerically by computational software, the most known being Zemax².

Commercially, two main strategies for building a passive and miniaturized focusing element exist: construct a GRIN lenses ${ }^{3}$ or assembly miniaturized achromatic doublets or triplets ${ }^{4}$. Numerical aperture, chromatic and geometric aberrations and their impact on image properties must be carefully considered during the modeling step regarding to the dimensions of the optical waveguide.

\section{Optical waveguide and scanning device}

A double clad fiber with a miniaturized scanning device at endoscopic distal end A double clad fiber (DCF) is an optical fiber composed by a single core which guides the excitation beam and two claddings, as illustrated in Fig. 2 (left). The inner cladding contributes to guide the excitation beam into the fiber core. The collection of the emission light is led by the inner cladding. Photonics crystal fiber (PCF) are microstructured fibers often chosen for their optimized guiding properties for both excitation delivering and emission collection [11]. With a single core fiber and in order to generate an image, a distal scanning device must be associated to the focusing element positioned at endoscopic distal end. This can be realized thanks to (i) a piezoelectric device [7] or (ii) a micro electromechanical mirror system (MEMS) [4, 7]. This last point, and the specificities associated to its miniaturization, constitutes the main technical difficulty of this strategy.

\footnotetext{
2 https://www.zemax.com/

3 https://www.grintech.de/en/

4 https://www.bernoptics.com/ or https://www.edmundoptics.com/
} 


\section{A fiber-optics bundle with a proximal galva-resonant scanning device}

A fiber-optics bundle is an optical waveguide composed by few thousands of fiber cores with a millimetric external diameter $[2,6]$. For an optimal light guiding and avoiding optical crosstalk, each core must be independent from its neighbor. That imposes optical properties of each core drastically different to each other and results in a significant difference in optical index between two neighbor cores. In that condition, each core of the bundle constitutes a pixel of the image. On an indicative basis, a usual image weakly resolved and generated with a bulk optical microscope is constituted by a square of 256 pixels $\times 256$ pixels which contains about 65000 pixels. An image generated with an endomicroscope based on a fiber-optics bundle is thus more weakly resolved than usual microscopic images. Moreover, parameters such as the distance between two cores, the core diameter and their relative homogeneity are impacting image quality: resolution and collection efficiency. This last point constitutes the main technical difficulty of this strategy. Thus, generating an exploitable image with a fiberoptics bundle is closely linked to a computational strategy erasing the impact of core inhomogeneities [5].

The main interest of a fiber-optics bundle concerns the scanning device. Indeed, each fiber core constituting the pixel of the image, the image is generated thanks to the sequential injection of the excitation beam into each core. Thus, the scanning device is positioned at the proximal input of the optical tube and does not require to be miniaturized. Many solutions of scanning devices are thus offered and do not impose any miniaturization: two galvanometric mirrors or a single galvanometric mirror combined with a resonant mirror for example.

Difference between a confocal and a multiphoton endomicroscopic system 
Similar physical principles are involved between confocal (or multiphoton) microscopy and confocal (or multiphoton) endomicroscopy. The main difference between microscopy and endomicroscopy concerns the delivering of the excitation laser light at the optical waveguide distal end. For confocal microscopy, no temporal constraint governs excitation beam. In that situation, no significant optical adjustments are required for confocal endomicroscopy except the use of an optical waveguide.

However, an essential adaptation of the optical excitation strategy must be developed in case of multiphoton endomicroscopy. Indeed, considering that a temporal confinement of excitation beam optimizes drastically the generation of multiphoton signals, the shortest pulse duration is favorable to an efficient generation of multiphoton processes. Thus, near infrared femtosecond pulses with a $\mathrm{MHz}$ repetition rate give a good solution for generating multiphoton processes. But when injected into an optical waveguide, linear and nonlinear effects appear and result in spectrotemporal distortions of pulse profile. Delivering femtosecond pulses at fiber output imposes a prechirp unit positioned at proximal input, before the endoscopic fiber [7, 14]. Prism compressor, grating stretcher, grisms line or chirp mirrors are few example of passive optical elements currently used to compensate for linear and nonlinear effects occurring into the core of the optical fiber. Many active solutions, involving active systems such as spatial light modulators are also sometimes used [g]. Such optical mask is the exclusive solution adapted with a fiber-optics bundle due to the drastically different dispersion properties between two neighbor cores.

\section{Biomedical Relevance}

In vivo and in situ imaging of the interior of organs allow hospital practitioners to gather information about anatomical structure and its relation to pathology. Structure or the 
metabolic state of organs become accessible in live and is of first interest for helping to orientate a medical diagnostic. An early diagnostic of cancers (lung or larynx for example) is associated to a procedure less painful when no surgical biopsy is required. Moreover in the context of preclinical researches, for spleen studies or neuroscience, the use of small animals and their ineluctable sacrifice is not a satisfying practice ethically. Furthermore, many information about metabolic states and evolutions become complex to identify in live, especially after the animal death. Resorting to endomicroscopy is an alternative solution which opens new opportunities in preclinical researches.

\section{Parameters of Image Quality}

\subsection{Label-free imaging of biological constituents}

Beyond the technical complexities specific to endomicroscopy, the endomicroscopic examination is also governed by the ability of the biological constituents to emit endogenously an optical signal. Several substances are known for their label-free fluorescence or for their ability to generate a second harmonic signal [8]. In the context of label-free imaging of biological substances, many alternative solutions of optical imaging are also currently on the spotlight. Fluorescence life time imaging (FLIM) and coherent anti-Stokes Raman scattering (CARS) are two examples of alternative solutions for generating an endogenous signal. However, their adaptation to endoscopy is even more complex technically than confocal or multiphoton fluorescence where the main limitation concerns the low level of emission flux difficult to separate from image noise. More information can be found about these two alternative techniques in chapter I. 8. a. FRET-FLIM and in chapter I. 9. f \& g Raman 
imaging \& Coherent Raman Imaging (CARS \& SERS). Endogenous fluorescence is also studied with alternative laser systems based on optical parametric oscillators [15] or ultrawide band laser systems [16]. Today, we could consider that the biological substances endogenously fluorescent have not yet been all characterized due to the lack of adapted laser system.

\subsection{Movements and endomicroscopic examination}

In vivo endomicroscopic imaging of living guinea pig is necessarily associated to body movements, at least due to breathing movements, and unavoidable in case of endomicroscopic imaging of small animal in living conditions. In that situation, the question of the optical waveguide fixation becomes of great importance and micrometric movements of the optical waveguide cannot be avoidable. Moreover, movements of the optical waveguide can also be associated to modifications of optical properties which impact the image quality and especially contrast and intensity levels. These external constraints are affecting precision and sensitivity of measurement. In case of double contrast agent, the geometric alignment and the fuse of an image pair becomes complex and imaging depth a high challenge. A computational strategy of image registration becomes indispensable [17]. This computational step estimates the geometric transformation and corrects the spatial superposition of the anatomical or functional structures imaged.

\section{Data Processing}

Data processing is key point for several steps of the image formation through an endomicroscopic system. First, depending on the optical waveguide chosen, the spiral oscillations in case of DCF or the pixel merging in case of a fiber-optic bundle entails 
the aid of a specific computational strategy for image reconstruction. Then, in case of movements of the optical waveguide, the image registration is indispensable and can also benefit from the strategies already developed for alternative medical imaging such as magnetic resonance imaging, positron emission tomography (PET) or optical coherence tomography (OCT).

\section{Conclusions}

\subsection{Strength \& Limitations}

The micrometric imaging of the interior of organs and imaging in live its metabolic state are of first interest for helping clinicians for an early diagnostic of diseases such as cancers or for the discrimination of healthy or cancerous tissues. Currently, the maturity of endomicroscopic systems may be no enough and is probably not yet well implanted into hospitals. Many reasons could explain this fact, starting from the habits of hospital practitioners. Technical constraints are also limiting image formation with an endomicroscopic system. For example, image field of view is limited to a square of 200 $\mu \mathrm{m}$ on each side. Moreover, only a few of biological substances can be imaged without labeling and the question of image registration stays significant. Considering these points, the use of a surgical biopsy, associated with external labelling and imaging with standard and bulk optical microscopes keeps the forefront place for guiding the research of a reliable diagnosis.

\subsection{Future Developments}

The future developments of endomicroscopy concern in fact many scientific and technological key points. Going to an endomicroscopic presenting (1) at least a millimetric field of view, (2) with a sub cellular resolution, (3) in millimetric depths, (4) 
with many endogenous signals could be realized in many years thanks to great progresses in: (i) miniaturization of optics with reduced optical aberrations, (ii) optimized optical waveguide, (iii) a new system for axial scanning, (iv) the characterization of endogenous signals from new substances of interest. More generally, the future developments of endomicroscopic systems rest on a general progress of technologic and scientific knowledges and discoveries, covering at least the fields of physics, chemistry, biology, informatics and mathematics.

\section{References \& Further Readings}

[1] Rousse A R, Gmitro A F 2000 Optics Letters 25 (23) 1708 - 1710

[2] Juskaitis R, Wilson T, Watson T F 1997 Scanning 1915 - 19

[3] Fu L, Gan X, Gu M 2005 Optics Express 13 (14), 5528 - 5534

[4] Myaing M T, MacDonald D J, Li X 2006 Optics Letters, 31 (8), 1076 - 1078

[5] Garofalakis A, Kruglik S G, Mansuryan T, Gillibert A, Thiberville L, Louradour F, Vever-Bizet C, Bourg-Heckly G 2019 J. Biomedical Optics, 24 (10), 106004

[6] Gu M, Bao H, Kang H 2014 Journal of Microscopy, 254 (1), 13 - 18

[7] Ducourthial G, Lefort C, Peyrot D A, Mansuryan T, Kruglik S, Vever-Bizet C, Thiberville L, Lacombe F, Bourg-Heckly G, Louradour F 2013, Proc SPIE, 8575

[8] Liang W X, Hall G, Messerschmidt B, Li M-J, Li X, 2017, Light, 6, e17082

[9] Lefort C, Hamzeh H, Louradour F, Pain F, Haidar DA, 2014 J. Biomedical Optics 19 (7), 076005

[10] Andreana M, Le T, Drexler W, Unterhuber A 2019 Opt. Letters, 44 (7) 1588 - 1591

[11] Lakowicz J R 2006 Principle of fluorescence spectroscopy, Third edition Springer [12] Fu L, Jain A, Xie H, Cranfield C, Gu M 2006 Optics Express, 14 (3), 1027 - 1032 [13] Fu L, Gu M 2007 Journal of Microscopy 226, 195 - 206 
[14] Lefort C, Kalashyan M, Ducourthial G, Mansuryan T, O'Connor RP, Louradour F 2014 JOSA B, 31 (10), $2317-2324$

[15] Tragardh J, Robb G, Amor R, Amos W B, Dempster J, McConnel G 2015 Journal of Microscopy, 259 (3) $210-218$

[16] Poudel C, Kaminski C, 2019 JOSA B, 36 (2), A139 - A153

[17] Rueckert D, Schnabel J A 2014 Registration and Segmentation in Medical Imaging, Springer 